\title{
Effects of coated cysteamine hydrochloride on muscle fiber characteristics and amino acid composition of finishing pigs
}

\author{
Miaomiao Bai ${ }^{1,2}$, Hongnan Liu ${ }^{1,3,4, *}$, Kang $\mathrm{Xu}^{1,3,4}$, Rong $\mathrm{Yu}^{3}$, Abimbola Oladele Oso ${ }^{5}$, \\ Jinping Deng ${ }^{2}$, and Yulong Yin ${ }^{1,2,3,4, *}$
}

\footnotetext{
* Corresponding Authors:

Hongnan Liu

Tel: +86-073184619761, Fax: +86-0731-84612685,

E-mail: liuhn@isa.ac.cn

Yulong Yin

Tel: +86-073184619761, Fax: +86-0731-84612685,

E-mail: yinyulong@isa.ac.cn

'Scientific Observing and Experimental Station of Animal Nutrition and Feed Science in South-

Central, Ministry of Agriculture, Hunan Provincial

Engineering Research Center for Healthy Breeding of Livestock and Poultry, Key Laboratory of Agro-

ecological Processes in Subtropical Region, National

Engineering Laboratory for Pollution Control and

Waste Utilization in Livestock and Poultry Production,

Institute of Subtropical Agriculture, Chinese Academy

of Sciences, Changsha 410125, China

${ }^{2}$ College of Animal Science, South China Agricultural

University, Guangzhou, Guangdong 510642, China

${ }^{3}$ Hangzhou King Techina Technology Company

Academician Expert Workstation, Hangzhou King

Techina Technology Co., Ltd., Hangzhou 311107,

China

${ }^{4}$ Hunan Co-Innovation Center of Animal Production

Safety, CICAPS, Changsha, Hunan 410128, China

${ }^{5}$ Department of Animal Nutrition, College of Animal

Science and Livestock Production, Federal University

of Agriculture, Abeokuta PMB 2240, Nigeria
}

ORCID

Miaomiao Bai

https://orcid.org/0000-0001-6984-0225

Hongnan Liu

https://orcid.org/0000-0002-5710-8624

Kang Xu

https://orcid.org/0000-0002-8568-1540

Rong Yu

https://orcid.org/0000-0001-6861-8737

Abimbola Oladele Oso

https://orcid.org/0000-0001-9107-0933

Jinping Deng

https://orcid.org/0000-0002-8964-9986

Yulong Yin

https://orcid.org/0000-0001-6112-6975

Submitted Jun 1, 2018; Revised Aug 15, 2018;

Accepted Sept 28, 2018
Objective: This experiment was designed to determine the effects of coated cysteamine hydrochloride (CC) on muscle fiber characteristics, amino acid composition and transporters gene expression in the longissimus dorsi muscle (LDM) of finishing pigs.

Methods: Two hundred and sixteen Duroc/Landrace/Yorkshire cross-bred male finishing pigs were fed with a corn-soybean basal diet supplemented with 0,70 , and $140 \mathrm{mg} / \mathrm{kg}$ cysteamine. Each group contained eight replicates of nine pigs per replicate. After 29 days, one pig was randomly selected from each replicate and slaughtered. Blood and LDM samples were collected and analyzed.

Results: The results showed that supplemental dietary CC increased $(\mathrm{p}<0.05)$ the muscle fiber density. And CC supplementation also up-regulated $(\mathrm{p}<0.05)$ the expression of myosin heavy chain 1 (MyHC1) and MyHC2x mRNA levels, and down-regulated ( $\mathrm{p}<0.05)$ MyHC2b expression in the LDM. Additionally, supplemental dietary CC reduced $(\mathrm{p}<0.05)$ the concentration of total cholesterol in the plasma and enhanced $(p<0.05)$ the concentrations of essential amino acid and total amino acid in the LDM. The relative expression levels of chloramphenicol acetyltransferase $2, \mathrm{~b}^{0,+}$ amino acid transporter, and $\mathrm{y}^{+}$-L-type amino acid transporter 1 were upregulated $(\mathrm{p}<0.05)$ in the LDM when pigs were fed with the dietary CC of $70 \mathrm{mg} / \mathrm{kg}$.

Conclusion: Cysteamine supplementation could increase fiber density and distribution of fiber types. It also improved the deposition of protein in the LDM by up-regulated the expression of amino acid transporters.

Keywords: Coated Cysteamine Hydrochloride; Finishing Pigs; Muscle Fiber; Amino Acids

\section{INTRODUCTION}

Meat quality is an important economic trait in meat industry, with the freshness and nutritional value of meat being the key factors that governs consumers' buying decision [1]. However, with the intensive selection for greater growth rate and lean percentage of pigs, meat quality also has deteriorated [2]. Therefore, identifying new ways to improve meat quality and nutritive value is important for human health and animal productivity. Meat quality is affected by the interaction between many factors such as muscle structure, postmortem environmental conditions, meat processing, packaging, and storage conditions, etc. [3]. Muscle fiber characteristics are the basic and direct factors to affect meat quality [4]. Muscle fiber composition is received to distinguish red and white muscles, which predicts biochemical changes and consequently meat quality [5].

Meat protein value has been assessed according to its amino acids composition. The amino acid composition is considered as an important index for nutritional value of each food. Numerous studies have verified that dietary supplementation with extra amino acids can influence the amino acid profile of tissues, particularly muscles [6]. Additionally, different 
amino acids are transported by specific amino acid transporters (neutral, acidic, or basic) in the muscle. The difference of quantitative expression of amino acid transports reflects the process of amino acid metabolism.

Cysteamine, as a novel feed additive in animal production, is used in the form of coated cysteamine hydrochloride (CC). Numerous researches have investigated its biological activity, which induces the regulation of mTOR signaling for modifying protein metabolism and antioxidative activities [6,7]. Using the commercial pig model, the previous study has shown supplemental dietary cysteamine improved the stability of pork color by regulating oxidation [8]. Cysteamine is a physiological performance promoter, which regulates protein synthesis and protein degradation in pigs [9]. The influence of cysteamine supplementation on protein digestion and absorption may be due to its improving jejunal amino acid transportation [10]. However, the effects of cysteamine on the regulation of amino acids metabolism in the muscle has not been investigated. The ability of cysteamine to enhance the muscle fiber characteristics of finishing pigs remains unexplored. A coating product prevents its dissolution or disintegration in the gastric environment. Therefore, based on these previous studies, it was hypothesized that supplementation with CC might influence muscle fiber characteristics, muscular deposition of protein and gene expression of transporters in the longissimus dorsi muscle (LDM) of finishing pigs thereby improving its meat quality and nutritive.

\section{MATERIALS AND METHODS}

\section{Animals and sampling}

The animal experiment protocol (NO. 20160712) was approved by the animal welfare committee of the Institute of Subtropical Agriculture, The Chinese Academy of Sciences, Changsha, China.

Two hundred and sixteen cross-bred (Duroc/Landrace/Yorkshire) castrated pigs with an average initial weight of $88.3 \pm 0.3$ $\mathrm{kg}$ were used in this study. Pigs were individually divided into three treatment groups, and each group was replicated eight times with nine pigs per replicate. The basal corn-soybean meal diet was respectively supplemented with 0 (control, $\mathrm{CON}$ ), $70 \mathrm{mg} / \mathrm{kg}$ (CC70), and $140 \mathrm{mg} / \mathrm{kg}$ (CC140) cysteamine supplied by coated CC (basal dietary composition is presented in Table 1). The basal diet was formulated to meet the nutritional requirement of finishing pigs recommended by the NRC [11]. Pigs had free access to feeds and drinking-water throughout the experimental stage. The experiment lasted for $29 \mathrm{~d}$. Additionally, coated CC, supplied by Hangzhou King Techina Technology Co., Ltd (Hangzhou, China), contained 270 g/kg CC.

After a 29-day period feeding experiment, one pig randomly selected from each pen was fasted overnight and the morning
Table 1. Composition and calculated nutrient levels of the basal diet (air-dry basis)

\begin{tabular}{|c|c|}
\hline Items & Content \\
\hline \multicolumn{2}{|l|}{ Ingredients (g/kg) } \\
\hline Corn (4.52\% crude protein) & 580.0 \\
\hline Soybean meal $(8.84 \%)$ & 200.0 \\
\hline Wheat bran & 80.0 \\
\hline Rice bran & 100.0 \\
\hline Vitamin premix ${ }^{11}$ & 20.0 \\
\hline Mineral premix & 20.0 \\
\hline Total & 1000 \\
\hline \multicolumn{2}{|l|}{ Nutrient content ${ }^{2)}$} \\
\hline Digestible energy (MJ/kg) & 13.12 \\
\hline Crude protein $(\%)$ & 16.40 \\
\hline Calcium (\%) & 0.80 \\
\hline Total phosphorus (\%) & 0.60 \\
\hline Available phosphorus (\%) & 0.40 \\
\hline Lysine $(\%)$ & 0.77 \\
\hline Methionine (\%) & 0.32 \\
\hline Methionine+cysteine (\%) & 0.60 \\
\hline \multicolumn{2}{|c|}{ 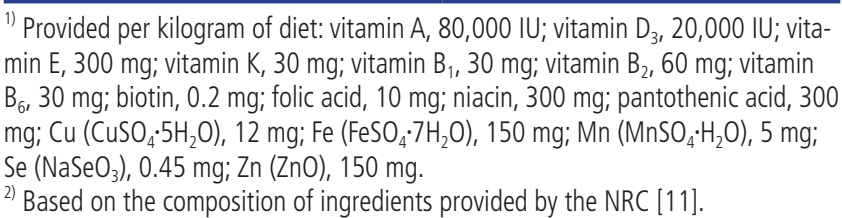 } \\
\hline
\end{tabular}

for $12 \mathrm{~h}$ and the killed by exsanguination after electrical stunning ( $250 \mathrm{~V}, 0.5 \mathrm{~A}$, for 5 to $6 \mathrm{~s}$ ). Fresh blood samples from external jugular vein were collected and kept in vacuum tubes with ethylenediaminetetraacetic acid. Plasma was obtained by centrifugation at $3,500 \times \mathrm{g}$ for 15 minutes at $4^{\circ} \mathrm{C}$, and the supernatant stored at $-20^{\circ} \mathrm{C}$ for analyzing biochemical indices. Muscle samples were carefully removed from the LDM at between 6th and 7th thoracic vertebrae within 45 min postmortem. The LDM samples were manually trimmed into $0.5 \times 0.5 \times 1.0$ $\mathrm{cm}$ pieces, promptly fixed in $10 \%$ formalin for microsection manufacture. Subsequently, about $20 \mathrm{~g}$ LDM samples were rapidly excised and weighted, then freeze-dried for amino acid analysis. Moreover, the extra LDM sample was packaged in foil, promptly frozen in liquid nitrogen, and stored at $-80^{\circ} \mathrm{C}$ for molecular analysis.

\section{Plasma biochemical indices}

Plasmas were centrifuged at $3,500 \times \mathrm{g}$ for 15 minutes at $4^{\circ} \mathrm{C}$, and the supernatants were used to determine biochemical indices by an Automatic Biochemical Analyzer (F. HoffmannLa Roche Ltd, Basel, Switzerland). These plasma biochemical indices, containing the concentrations of total protein, triglyceride, total cholesterol, glucose, urea nitrogen, and the activities of aspartate aminotransferase, alanine aminotransferase, and alkaline phosphatase, were analyzed according to the commercial reagents form Roche Diagnostics $\mathrm{GmbH}$ (Roche Diagnostics GmbH, Mannheim, Germany) and manufacturer's instructions [12]. 
Muscle fiber characteristics measurement

Muscle fiber density and diameter were measured by the traditional staining of hematoxylin and eosin (HE). Samples of the LDM were transferred form $4 \%$ paraformaldehyde to $70 \%$ ethanol. Before dehydrated through a serial alcohol gradient, individual sections of muscle biopsy material were placed in processing cassettes. The sections embedded into paraffin wax blocks were cut into $5 \mu \mathrm{m}$ thickness and dewaxed in xylene, then rehydrated through lower concentrations of ethanol. After washed in phosphate buffer saline, sections were stained with $\mathrm{HE}$ and dehydrated through increasing concentrations of ethanol and xylene. The morphology of the LDM was observed at 200 times under a light microscope [13]. The Image-pro plus-6.0 pathologica system was applied for imaging analysis, and three views were selected for each section. Muscle fiber characteristics were determined by measuring muscle fiber density and diameter. Approximately 10 views were counted to estimate the cross-sectional area of the muscle fiber. Muscle fiber density was expressed as the ratio of total number of fibers counted to the total area of muscle fiber measured. Muscle fiber diameters were analyzed using the Image-Pro plus 5.1, which was an image analysis system (Media Cybernetics Inc., Rockville, MD, USA) [14].

Amino acid profile in muscle

Amino acid compositions (hydrolytic amino acids) of the LDM were determined by an amino acid analyzer (L8900, Hitachi, Tokyo, Japan). Amino acids were extracted from tissue following the technique described by $\mathrm{Hu}$ et al [15] with slight modifications. About $0.1 \mathrm{~g}$ of freeze-dried muscle samples was hydrolyzed with $10 \mathrm{~mL}$ of hydrochloric acid solution $(\mathrm{HCl}, 6 \mathrm{M})$ in a sealed ampoule bottle for $22 \mathrm{~h}$ at $110^{\circ} \mathrm{C}$. Then all hydrolysate was diluted with double-distilled water in volumetric flask of $100 \mathrm{~mL}$, subsequently $1 \mathrm{~mL}$ of hydrolysate diluent was passed through a $0.22 \mathrm{~mm}$ membrane filter. Samples were analyzed for amino acids contents through comparing peak profiles of the squid samples with standard amino acid profiles.

\section{Real-time quantitative polymerase chain reaction analysis}

Total RNA was extracted from LDM samples using a Trizol Reagent (Invitrogen, Carlsbad, CA, USA) following manufacturer's instructions and dissolved in diethylpyrocarbonate (DEPC)-treated water. The extracted RNA was quantified by measuring optical density at 260 and $280 \mathrm{~nm}$ using an Eppendorf Biophotometer (Eppendorf AG, Hamburg, Germany) and its integrity verified by electrophoresis on a $1 \%$ agarose gel. The cDNA was reverse-transcribed from $1 \mu \mathrm{g}$ of total RNA using the DNase I treatment (Takara, Otsu, Japan), then diluted and used for evaluating gene expression. All primer sequences employed herein were developed previously for amplification of mRNA sequences of Sus scrofa (Table 2). These target genes

Table 2. Primers used for quantitative real-time polymerase chain reaction

\begin{tabular}{|c|c|c|c|c|}
\hline Gene & Accession No. & Primer, 5'-3' & Size (bp) & $\mathrm{T}_{\mathrm{A}}\left({ }^{\circ} \mathrm{C}\right)$ \\
\hline \multirow[t]{2}{*}{ GAPDH } & NM_001206359.1 & F: ACTCACTCTTCTACCTTTGATGCT & & \\
\hline & & R:TGTTGCTGTAGCCAAATTCA & 100 & 59 \\
\hline \multirow[t]{2}{*}{$\mathrm{MyHCl}$} & NM_001190422 & F: AAGGGCTTGAACGAGGAGTAGA & & \\
\hline & & R: TTATTCTGCTTCCTCCAAAGGG & 114 & 57 \\
\hline \multirow[t]{2}{*}{$\mathrm{MyHC2a}$} & NM_214136.1 & F: GCTGAGCGAGCTGAAATCC & & \\
\hline & & R:ACTGAGACACCAGAGCTTCT & 137 & 58 \\
\hline \multirow[t]{2}{*}{$\mathrm{MyHC2b}$} & NM_001123141.1 & F:ATGAAGAGGAACCACATTA & & \\
\hline & & R:TTATTGCCTCAGTAGCTTG & 166 & 52 \\
\hline \multirow[t]{2}{*}{$\mathrm{MyHC2x}$} & NM_001104951.1 & F: AGAAGATCAACTGAGTGAACT & & \\
\hline & & R:AGAGCTGAGAAACTAACGTG & 149 & 55 \\
\hline \multirow[t]{2}{*}{ ASCT2 } & XM_003127238.1 & F: GATTGTGGAGATGGAGGATGTGG & & \\
\hline & & R: TGCGAGTGAAGAGGAAGTAGATGA & 128 & 60 \\
\hline \multirow[t]{2}{*}{ CAT1 } & NM_001012613.1 & F:TCTGGTCCTGGGCTTCATAA & & \\
\hline & & R:ACCTTCGTGGCATTGTTCAG & 123 & 58 \\
\hline \multirow[t]{2}{*}{ CAT2 } & NM_001110420.1 & F:ACAACTGGCGAAGAAGTCCG & & \\
\hline & & R: CTGCCGAGACCCCAAAATAG & 100 & 60 \\
\hline \multirow[t]{2}{*}{$y^{+}$LAT1 } & XM_013978228.1 & F: GAGTGCCAGAACACAAACGA & & \\
\hline & & R:TCCTCCATCTTCCAAATCCA & 116 & 58 \\
\hline \multirow[t]{2}{*}{ LAT2 } & XM_011978238.1 & F: CACCATTCCCTGGCTACTCT & & \\
\hline & & R: TCCTACCACTGCCTGACAAA & 185 & 58 \\
\hline \multirow[t]{2}{*}{$b^{0,+} A T$} & NM_001110171.1 & F: GCCTATCAAGGTGCCCATC & & \\
\hline & & R:AGCGGACGAACAGGAAGTAA & 155 & 59 \\
\hline
\end{tabular}

$\mathrm{T}_{A}$, annealing temperature; GAPDH, glyceraldehyde-3-phosphate dehydrogenase; MyHC, myosin heavy chain; ASCT2, autologous stem cell transplantion 2; CAT, chloramphenicol acetyltransferase; $y+L A T 1, y^{+}$-L-type amino acid transporter $1 ; L A T 2, L$-type amino acid transporter $2 ; b 0,+A T, b^{0,+}$ amino acid transporter. 
respectively includes muscle fiber types (myosin heavy chain 1 [MyHC-1], 2a, 2b, and 2x), and amino acid transporters (autologous stem cell transplantion 2 [ASCT2], chloramphenicol acetyltransferase [CAT]-1 and 2, $\mathrm{y}^{+}$-L-type amino acid transporter [y $\left.\mathrm{y}^{+} \mathrm{LAT} 1\right]$, LAT2, and $\mathrm{b}^{0,+}$ amino acid transporter $\left.\left[\mathrm{b}^{0,+} \mathrm{AT}\right]\right)$. Amplification of the housekeeping gene (glyceraldehyde3-phosphate dehydrogenase) and target genes was performed in a $10-\mu \mathrm{L}$ reaction volume including $1 \mu \mathrm{M}$ of each forward and reverse primer, $2 \mu \mathrm{L}$ of cDNA, $2 \mu \mathrm{L}$ of DEPC-treated water, and $5 \mu \mathrm{L}$ of SYBR Premix Ex Taq (Takara Bio Inc., Japan). The relative expression levels of genes were performed using Lightcycler-480II system (Roche Diagnostics GmbH, Germany). The real-time polymerase chain reaction (RT-PCR) protocol was strictly carried out with forty cycles of amplification, with each cycle consisting of pre-denaturation at $95^{\circ} \mathrm{C}$ for $10 \mathrm{~s}$; denaturation at $95^{\circ} \mathrm{C}$ for $5 \mathrm{~s}$; annealing at $60^{\circ} \mathrm{C}$ for $30 \mathrm{~s}$; and elongation at $52^{\circ} \mathrm{C}$ to $60^{\circ} \mathrm{C}$ for $30 \mathrm{~s}$, followed by a melting curve analysis. Cycle threshold $(\mathrm{Ct})$ values were employed to determine expression levels of genes and calculated of triplicate measurements. The mRNA expression levels were analyzed and expressed as the relative values to those for control pigs. The comparative Ct value method was descried by Tan et al [16].

\section{Statistical analysis}

The experimental data were analyzed statistically by the one- way analysis of variance method using SAS (SAS Institute Inc., Cary, NC, USA; 2002) and expressed as means \pm standard error of the mean. Based on adjusted degrees of freedom solution, this data about muscle fiber characteristics measurements and amino acid composition were calculated using mixed procedure for repeated measures. Duncan's multiple-range test was performed for indicating differences between significant mean values. The statistical differences were declared significant at $\mathrm{p}<0.05$ in all analyses.

\section{RESULTS}

\section{Plasma biochemical indices}

Plasma biochemical indices in finishing pigs were tested and shown in Table 3. CC70 markedly reduced $(\mathrm{p}<0.05)$ the concentration of total cholesterol in the plasma when compared with the control and CC140 groups. However, compared with CC140 group, supplemental dietary $70 \mathrm{mg} / \mathrm{kg}$ CC significantly reduced $(\mathrm{p}<0.05)$ alanine aminotransferase activity in the plasma.

\section{Muscle fiber characteristics}

The morphology of LDM fiber is shown in Figure 1. Based on fiber counting methods, the muscle fiber characteristics of finishing pigs in each group were measured and the data were

Table 3. Effects of dietary coated cysteamine hydrochloride (CC) on plasma biochemical indices in finishing pigs $(n=8)$

\begin{tabular}{|c|c|c|c|c|}
\hline \multirow{2}{*}{ Items } & \multicolumn{3}{|c|}{ Dietary level of $\mathrm{CC}^{1)}$} & \multirow{2}{*}{ p-value } \\
\hline & CON & CC70 & CC140 & \\
\hline Total protein (mmol/L) & $69.84 \pm 2.34$ & $72.20 \pm 1.74$ & $72.78 \pm 2.74$ & 0.644 \\
\hline Triglyceride (mmol/L) & $0.22 \pm 0.15$ & $0.17 \pm 0.09$ & $0.21 \pm 0.09$ & 0.593 \\
\hline Total cholesterol (mmol/L) & $2.18 \pm 0.07^{b}$ & $1.86 \pm 0.09^{\mathrm{a}}$ & $2.09 \pm 0.04^{b}$ & 0.015 \\
\hline Glucose (mmol/L) & $4.69 \pm 0.10$ & $4.86 \pm 0.15$ & $5.23 \pm 0.23$ & 0.097 \\
\hline Urea (mmol/L) & $7.47 \pm 0.37$ & $7.73 \pm 0.47$ & $6.62 \pm 0.67$ & 0.313 \\
\hline Alkaline phosphatase (U/L) & $142.45 \pm 11.96$ & $124.55 \pm 8.85$ & $113.76 \pm 10.91$ & 0.174 \\
\hline Alanine aminotransferase (U/L) & $50.76 \pm 1.80^{\mathrm{ab}}$ & $52.54 \pm 2.77^{b}$ & $44.04 \pm 2.47^{\mathrm{a}}$ & 0.048 \\
\hline Aspartate aminotransferase (U/L) & $38.15 \pm 2.43$ & $46.86 \pm 3.66$ & $40.15 \pm 11.98$ & 0.211 \\
\hline
\end{tabular}

1) Con, finishing pigs fed a basal diet containing $0 \mathrm{mg} / \mathrm{kg}$ coated cysteamine hydrochloride; CC70, finishing pigs fed a basal diet containing $70 \mathrm{mg} / \mathrm{kg}$ coated cysteamine hydrochloride; CC140, finishing pigs fed a basal diet containing $140 \mathrm{mg} / \mathrm{kg}$ coated cysteamine hydrochloride.

$a, b$ Mean values with unlike letters were significantly different $(p<0.05)$.
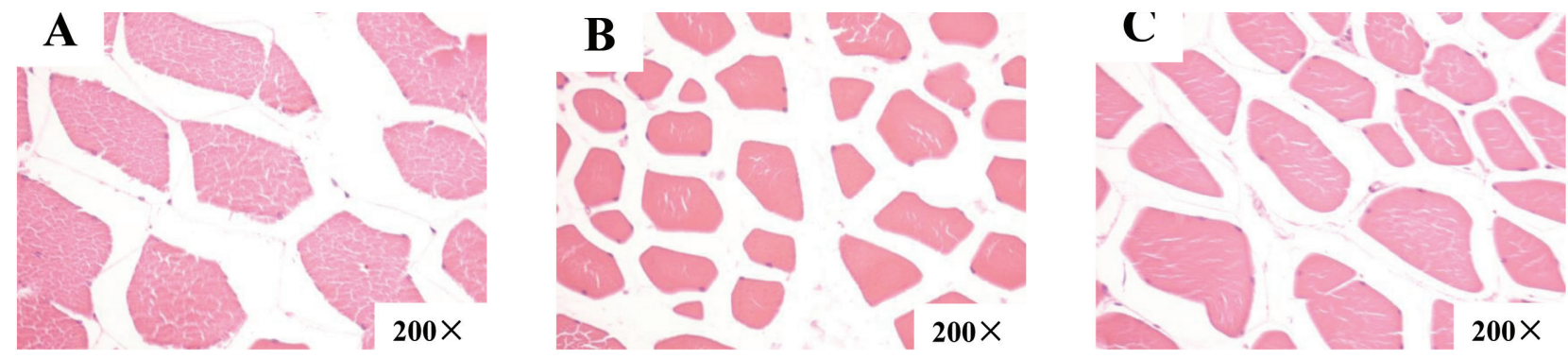

Figure 1. Morphology of longissimus dorsi muscle (LDM) fiber (200x). (A) Morphology of LDM fiber in pigs fed a basal diet containing 0 (CON). (B) Morphology of LDM fiber in pigs fed a basal diet containing $70 \mathrm{mg} / \mathrm{kg}$ (CC70). (C) Morphology of LDM fiber in pigs fed a basal diet containing $140 \mathrm{mg} / \mathrm{kg}$ (CC140). 
Table 4. Effect of dietary coated cysteamine hydrochloride (CC) on muscle fiber characteristics $(n=8)$

\begin{tabular}{|c|c|c|c|c|}
\hline \multirow{2}{*}{ Items ${ }^{2)}$} & \multicolumn{3}{|c|}{ Dietary level of CC $^{1)}$} & \multirow{2}{*}{$\mathrm{p}$-value } \\
\hline & CON & CC70 & CC140 & \\
\hline Fiber diameter ( $\mu \mathrm{m})$ & $59.1 \pm 0.91$ & $56.67 \pm 1.83$ & $59.54 \pm 0.96$ & 0.264 \\
\hline Fiber density $\left(n / \mu m^{2}\right)$ & $241.17 \pm 12.30^{a}$ & $284.45 \pm 9.86^{b}$ & $249.04 \pm 9.69^{a}$ & 0.013 \\
\hline
\end{tabular}

1) CON, finishing pigs fed a basal diet containing $0 \mathrm{mg} / \mathrm{kg}$ coated cysteamine hydrochloride; CC70, finishing pigs fed a basal diet containing $70 \mathrm{mg} / \mathrm{kg}$ coated cysteamine hydrochloride; CC140, finishing pigs fed a basal diet containing $140 \mathrm{mg} / \mathrm{kg}$ coated cysteamine hydrochloride.

2) Muscle fiber diameter and density were calculated by total number of fibers and cross-sectional area of fibers.

${ }^{a, b}$ Mean values with unlike letters were significantly different $(p<0.05)$.

analyzed, as shown in Table 4 . In the same field of vision, there were more muscle fibers in the LDM in pigs fed the CC70 diet. Compared to control treatment and CC140, CC70 had a higher muscle fiber density $(\mathrm{p}<0.05)$ in the LDM.

Gene expression levels of myosin heavy chain

As shown in Figure 2, effects of dietary CC supplementation on the mRNA level of MyHC isoform gene of finishing pigs were assessed via RT-PCR. Supplemental dietary $70 \mathrm{mg} / \mathrm{kg}$ CC significantly up-regulated $(\mathrm{p}<0.05)$ muscle $\mathrm{MyHC1}$ and $\mathrm{My}-$ HC2x mRNA levels. Meanwhile, compared to control group, a decreased muscle MyHC2b mRNA level $(\mathrm{p}<0.05)$ was observed with dietary $70 \mathrm{mg} / \mathrm{kg}$ CC supplementation. And the expression level of MyHC2a was lower $(p<0.05)$ in pigs fed control and CC70 than CC140 diet.

\section{Amino acid profile in muscle}

The amino acid profile that indicates protein metabolism in the LDM is detailed in Table 5. The concentrations of essential amino acid and total amino acid (TAA) were calculated based on the analyzed muscle amino acid contents in this study. Compared with CC140, supplemental dietary $70 \mathrm{mg} / \mathrm{kg}$ CC markedly improved $(\mathrm{p}<0.05)$ the muscle TAA. Leucine, isoleucine and valine are known as branched-chain amino acids. A significant increased isoleucine $(\mathrm{p}<0.05)$ was observed with pigs fed CC70 diet. Moreover, compared to control group, CC70
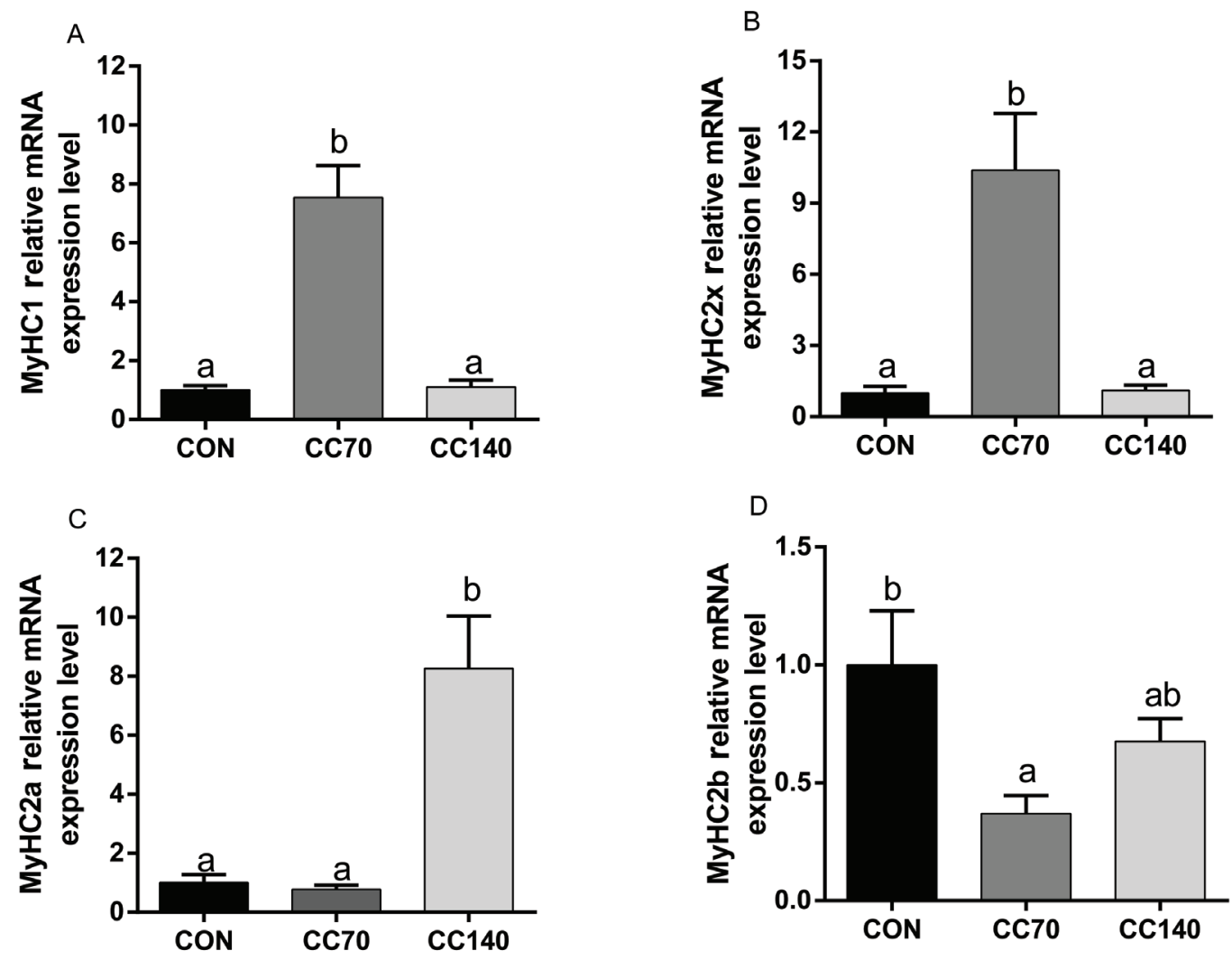

Figure 2. Expression levels of muscle fiber types of finishing pigs fed a basal diet containing 0 (CON), 70 (CC70) and $140 \mathrm{mg} / \mathrm{kg}$ (CC140) coated cysteamine hydrochloride, respectively. The figure indicates: (A) MyHC1; (B) MyHC2x; (C) MyHC2a; (D) MyHC2b. The error bars represents standard error. Mean values with unlike letters $(a, b)$ were significantly different $(p<0.05)$. 
Table 5. Effects of dietary coated cysteamine hydrochloride (CC) on amino acid composition in the longissimus dorsi muscle of finishing pigs $(n=8)$

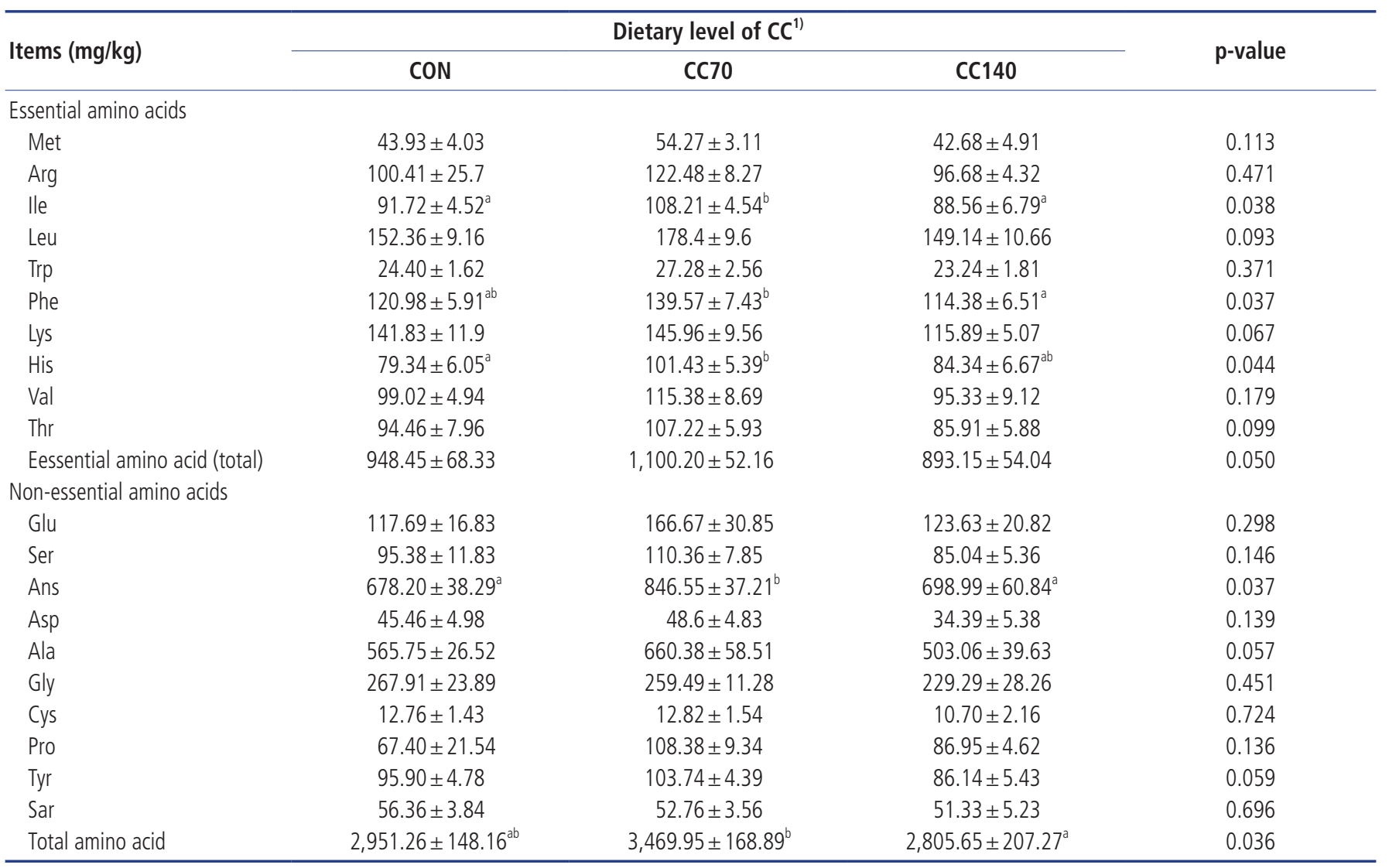

1) CON, finishing pigs fed a basal diet containing $0 \mathrm{mg} / \mathrm{kg}$ coated cysteamine hydrochloride; CC70, finishing pigs fed a basal diet containing $70 \mathrm{mg} / \mathrm{kg}$ coated cysteamine hydrochloride; CC140, finishing pigs fed a basal diet containing $140 \mathrm{mg} / \mathrm{kg}$ coated cysteamine hydrochloride.

$a, b$ Mean values with unlike letters were significantly different $(p<0.05)$.

significantly enhanced $(\mathrm{p}<0.05)$ the concentration of histidine in the LDM. In the neutral amino acids, CC70 increased $(\mathrm{p}<$ $0.05)$ the content of phenylalanine. Dietary $70 \mathrm{mg} / \mathrm{kg}$ CC supplemented pigs had higher $(\mathrm{p}<0.05)$ asparagine content in the LDM than control and $140 \mathrm{mg} / \mathrm{kg} \mathrm{CC}$.

Gene expression levels of amino acid transporters

To further explore the different influences of dietary CC on muscle amino acid composition, we measured the expression of key amino acid transporters The relative mRNA expression levels of CAT2, $\mathrm{y}^{+} \mathrm{LAT} 1$ and $\mathrm{b}^{0,+} \mathrm{AT}$ were up-regulated $(\mathrm{p}<0.05)$ by $70 \mathrm{mg} / \mathrm{kg}$ CC supplementation of the diet compared to the control diet (Figure 3C, 3D, 3F). However, no significant difference $(p>0.10)$ was observed on ASCT2, CAT1, and LAT2 expression in the LDM between groups (Figure 3A, 3B, 3E).

\section{DISCUSSION}

In livestock production dietary supplementation with certain nutrients is supposed to be an effective way of improving meat quality and nutrition [17]. Numbers of studies in vivo and in vitro have focused on the growth-promoting and antioxidant functions of cysteamine $[18,19]$. However, the previous studies have demonstrated that supplemental dietary CC had no significant effect on meat $\mathrm{pH}$ and drip loss, but increased meat color values and the deoxymyoglobin content by improving glutathione levels and antioxidant activity [20]. Meanwhile, this present study is first to indicate that dietary cysteamine supplementation can improve meat quality of finishing pigs through changing muscle fiber density and type distribution and promoting amino acids deposition and transportation.

Muscle fiber, as an important component of muscle, directly affects meat quality. Muscle fiber characteristics have been correlated with various meat quality traits including muscle $\mathrm{pH}$, tenderness, drip loss, meat color, and intramuscular fat content [21]. Moreover, muscle fiber number, size and fiber type composition are closely interrelated and were implicated in muscle fiber characteristics [22]. The fiber density was positively related to tenderness of cooked pork $[23,24]$. In the present study, dietary CC supplementation increased muscle fiber density in finishing pigs which indicates improving the sensory quality of meat.

Additionally, muscle fiber types can determine the size, number and density of muscle fiber. In general, muscle fibers are 

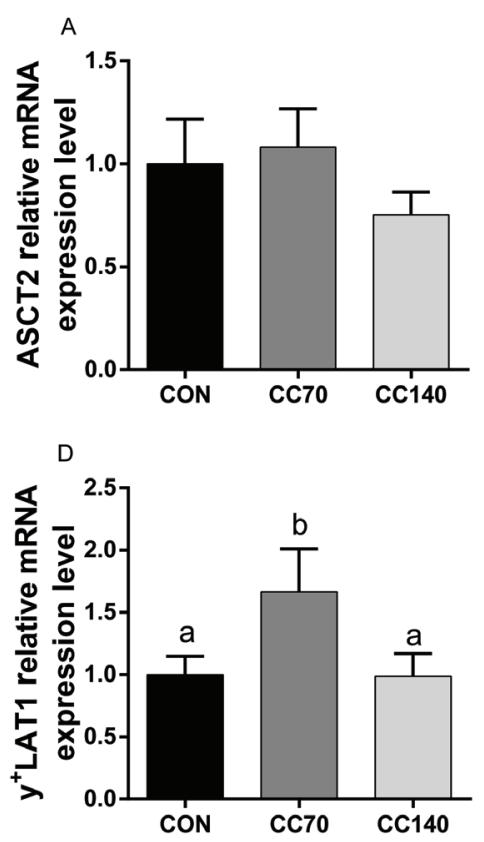
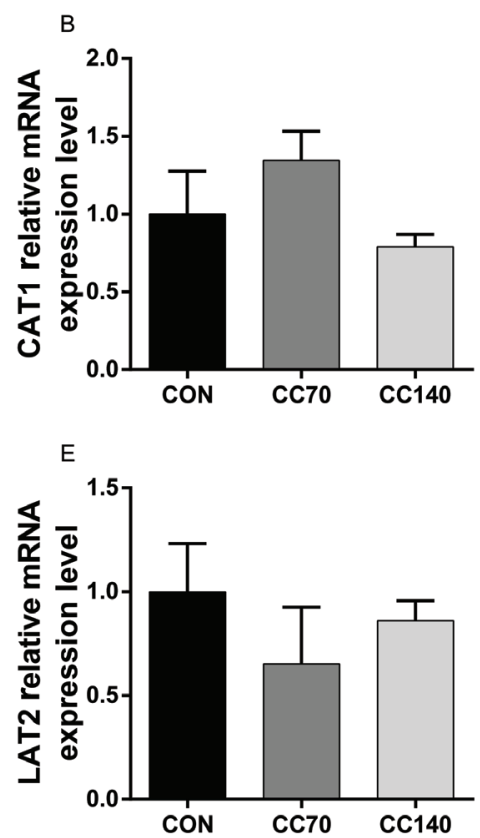
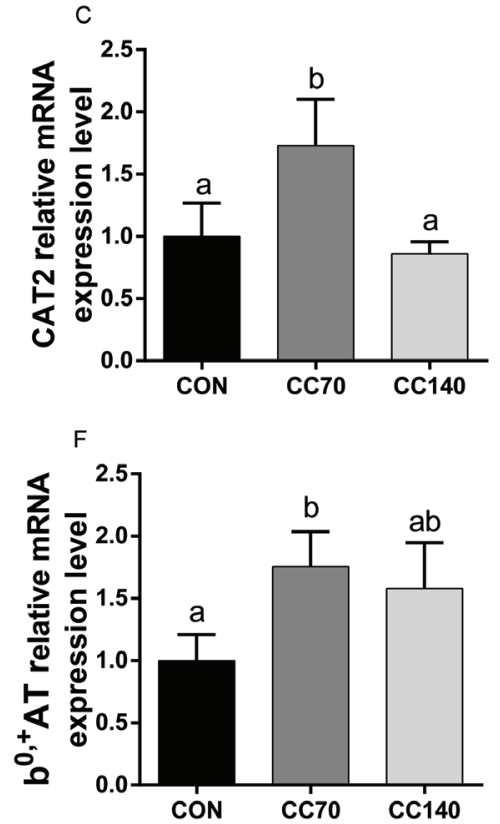

Figure 3. Effects of dietary coated cysteamine hydrochloride (CC) on the relative mRNA expression levels of ASC amino-acid transporter 2 (ASCT2), cationic amino acid transporter (CAT)-1, CAT2, L-type amino acid transporter (LAT)-2, $y^{+}$LAT1, and $b^{0,+}$ amino acid transporter $\left(b^{0,+} A T\right)$ in $(A, B, C, D, E$ and $F)$ the longissimus dorsi muscle of finishing pigs. The figure indicates which pigs were fed with a corn-soybean diet containing the CCH of 0 (CON), 70 (CC70), and $140 \mathrm{mg} / \mathrm{kg}$ (CC140), respectively. The error bars represents standard error. Mean values with unlike letters $(a, b)$ were significantly different $(p<0.05)$.

divided into four types considered as slow-oxidative type I, fast oxido-glycolytic type IIA, and fast glycolytic types IIX and IIB, and expressed by $\mathrm{MyHC}$ isoforms genes identified as $\mathrm{MyHC1}$, MyHC2a, MyHC2b, and MyHC2x [25]. According to Kim et al [26], the content of myoglobin and fiber color increases in the rank order I $>$ IIA $>$ IIX $>$ IIB, but the fiber size is in the opposite order. A higher type 1 fiber proportion might be positively correlated with muscle fiber density. Previous researches also reported that low percentages of type $2 \mathrm{~A}$ and $2 \mathrm{~B}$ fibers in the meat resulted in increased lightness $\left(\mathrm{L}^{*}\right)$ and redness $\left(\mathrm{a}^{*}\right)$. This is agreed with our previous study, which suggested that pigs fed dietary CC had increased $\mathrm{a}^{*}$ in the LDM which promoted the stability of pork color [19]. This was related to the myoglobin content of different fiber types. Furthermore, it has been demonstrated that antioxidant status is positively correlated with $\mathrm{a}^{*}$ or color stability in the LDM of beef [27]. Cysteamine, as an excellent scavenger of oxidants hydroxyl radical and hypochlorous acid, can protect against body oxidation. A muscle fiber type transition toward more oxidative muscle fibers such as $\mathrm{MyHC1}$ can be induced by antioxidant [28]. This present study firstly provides the results that dietary CC supplementation improves $\mathrm{MyHC1}$ and $\mathrm{MyHC} 2 \mathrm{x}$ expression and decreases MyHC2b mRNA expression, which may partly explain the mechanism of cysteamine on the improvement of meat quality.

Intramuscular amino acid composition and metabolism are determined to evaluate the biological value of muscle protein nutrition contributing to meat quality. This present study indicated that dietary CC supplementation enhances the mus- cle amino acids deposition of finishing pigs. Consistent with previous studies, cysteamine supplementation resulted in a marked stimulation of protein deposition in skeletal muscle $[6,29]$. Importantly, plasma metabolism profile is reflected by changes in physiological and metabolic activities in response to dietary manipulations [30]. In the present study, serum total cholesterol was decreased, and glucose was increased by dietary supplemented with CC, indicating the inhibition of lipid metabolism and acceleration of glucose metabolism. Cysteamine could accelerate glucose and amino acid into muscle for protein synthesis, which may be associated with an increase in insulin-like growth factor 1 consequently stimulating protein synthesis [31].

Additionally, specific transporters plays an important role for transporting amino acids into the cell and in response to amino acid availability [32]. Previous studies have found that amino acid transporters changed in small intestine, skeletal muscle, and other tissues once dietary nutrient content was varied [33]. At present, little information about the impact of cysteamine on amino acid transporters in the LDM is available. However, the branched-chain amino acids (Ile), alkaline amino acids (His) and neutral amino acids (Phe and Ans) contents were increased in the LDM in the present study. Different amino acid transporters unquestionably play a critical role in transportation with different mechanisms and featuring with unique substrate specificities [34]. The cationic amino acid transporters (CAT) have an important role in the transportation of arginine, lysine, histidine, and ornithine to regulate 
their homeostasis, and are widely distributed in tissues [35]. The ASCT2, $b^{0,+}$ AT, $y^{+}$LAT1, and LAT2 are neutral amino acids transporters, and are mainly involved in the transport of branched-chain amino acids and some small neutral amino acids including asparagine and glutamine [36]. In addition, $\mathrm{b}^{0,+}$ AT, and $\mathrm{y}^{+} \mathrm{LAT} 1$ are $\mathrm{Na}^{+}$-independent transporters [37]. This agreed with Zhou et al [10], who reported that cysteamine supplementation in finishing pigs enhanced protein digestion and absorption via upregulating the expression of amino acid transporters ( $\mathrm{y}^{+} \mathrm{LAT} 1$ and $\left.\mathrm{b}^{0,+} \mathrm{AT}\right)$ in the jejunum. Thus, it is possible that there is an inevitable correlation between muscle and intestine for transportation and absorption of nutrition. In addition, the present study has demonstrated that administration of $70 \mathrm{mg} / \mathrm{kg} \mathrm{CC}$ had a greater influence on muscle fiber characteristics and amino acid metabolism than $140 \mathrm{mg} / \mathrm{kg}$ CC supplemented in finishing pigs. This might be relative to the fact that continuous addition of a high dose of CC can cause gastrointestinal ulcer [38].

\section{CONCLUSION}

Dietary coated cysteamine increased the fiber density, up-regulated the expression levels of $\mathrm{MyHC1}$ and $\mathrm{MyHC} 2 \mathrm{x}$ and downregulated expression of $\mathrm{MyHC} 2 \mathrm{~b}$. In addition, coated cysteamine supplementation improved the deposition of amino acids by promoting amino acid transporters expression in the LDM. Above all this study presented the most appropriate dose of dietary $70 \mathrm{mg} / \mathrm{kg}$ CC supplementation, which will be useful in livestock industry to improve meat quality and nutritive value. Importantly, this study adds to the knowledge of cysteamine function but further research about underlying mechanisms are required.

\section{CONFLICT OF INTEREST}

We certify that there is no conflict of interest with any financial organization regarding the material discussed in the manuscript. Liu H, Xu K, Yin Y are employees of Hangzhou King Techina Technology Co., Ltd, and Yu R, Yin Y are employees of CICAPS.

\section{ACKNOWLEDGMENTS}

This work was supported by the National Natural Science Foundation of China (31501964 and 31872985), Youth Innovation Team Project of ISA, CAS (2017QNCXTD_TBE) and China Agriculture Research System (CARS-35). The research was also funded by a research program of the State Key Laboratory of Food Science and Technology and Key Research and Development program of Shandong province (2015STS007). We thank Hangzhou King Techina Technology Company Academician Expert Workstation and Shandong Newhope-Liuhe
Group Company Academicion Expert Workstation for providing technical assistance.

\section{REFERENCES}

1. Grunert KG, Bredahl L, Brunsø K. Consumer perception of meat quality and implications for product development in the meat sector-a review. Meat Sci 2004;66:259-72.

2. Lefaucheur L, Milan D, Ecolan P, et al. Myosin heavy chain composition of different skeletal muscles in large white and meishan pigs. J Anim Sci 2004;82:1931-41.

3. Joo ST, Kim GD, Hwang YH, et al. Control of fresh meat quality through manipulation of muscle fiber characteristics. Meat Sci 2013;95:828-36.

4. Lee S, Joo S, Ryu Y. Skeletal muscle fiber type and myofibrillar proteins in relation to meat quality. Meat Sci 2010;86:166-70.

5. Kim GD, Yang HS, Jeong JY. Intramuscular variations of proteome and muscle fiber type distribution in semimembranosus and semitendinosus muscles associated with pork quality. Food Chem 2018;244:143-52.

6. Zhou P, Zhang L, Li J, et al. Effects of dietary crude protein levels and cysteamine supplementation on protein synthetic and degradative signaling in skeletal muscle of finishing pigs. Plos One 2015;10:e0139393.

7. Zhang ZY, Yang MF, Wang T, et al. Cysteamine alleviates early brain injury via reducing oxidative stress and apoptosis in a rat experimental subarachnoid hemorrhage model. Cell Mol Neurobiol 2015;35:543-53.

8. Bai MM, Liu HN, Xu K, et al. Effects of dietary coated cysteamine hydrochloride on pork color in finishing pigs. J Sci Food Agric 2017;48:96-100.

9. Liu G, Wang Z, Wu D, et al. Effects of dietary cysteamine supplementation on growth performance and whole-body protein turnover in finishing pigs. Livest Sci 2009;122:86-9.

10. Zhou P, Luo Y, Zhang L, et al. Effects of cysteamine supplementation on the intestinal expression of amino acid and peptide transporters and intestinal health in finishing pigs. Anim Sci J 2017;88:314-21.

11. NRC. Nutrient requirements of swine. Washington DC, USA: National Academy Press; 2012 .

12. Deng J, Wu X, Bin S, et al. Dietary amylose and amylopectin ratio and resistant starch content affects plasma glucose, lactic acid, hormone levels and protein synthesis in splanchnic tissues. J Anim Physiol Anim N 2010;94:220-6.

13. Turek Z, Grandtner M, Kreuzer F. Cardiac hypertrophy, capillary and muscle fiber density, muscle fiber diameter, capillary radius and diffusion distance in the myocardium of growing rats adapted to a simulated altitude of $3500 \mathrm{~m}$. Pflügers Archiv 1972;335:19-28.

14. Qin PY, Ding YF, Xiao JH, et al. Effects of a traditional Chinese medicine formula and its extraction on muscle fiber characteristics in finishing pigs, porcine cell proliferation and isoforms 
of myosin heavy chain gene expression in myocytes. AsianAustralas J Anim 2017;30:1620-32.

15. Hu CJ, Jiang QY, Zhang T, et al. Dietary supplementation with arginine and glutamic acid modifies growth performance, carcass traits, and meat quality in growing-finishing pigs. J Anim Sci 2017;95:2680-9.

16. Tan B, Yin Y, Liu Z, et al. Dietary l-arginine supplementation differentially regulates expression of lipid-metabolic genes in porcine adipose tissue and skeletal muscle. J Nutr Biochem 2011;22:441-5.

17. Zhang C, Luo J, Yu B, et al. Dietary resveratrol supplementation improves meat quality of finishing pigs through changing muscle fiber characteristics and antioxidative status. Meat Sci 2015; 102(Supplement C):15-21.

18. Yang CB, Li AK, Yin YL, et al. Effects of dietary supplementation of cysteamine on growth performance, carcass quality, serum hormones and gastric ulcer in finishing pigs. J Sci Food Agric 2005;85:1947-52.

19. Barnett M, Hegarty R. Cysteamine: a human health dietary additive with potential to improve livestock growth rate and efficiency. Anim Prod Sci 2016;56:1330-8.

20. Bai MM, Liu HN, Xu K, et al. Effects of dietary coated cysteamine hydrochloride on pork color in finishing pigs. J Sci Food Agric 2018;98:1743-50.

21. Yu Q, Wu W, Tian X, et al. Unraveling proteome changes of holstein beef $\mathrm{m}$. Semitendinosus and its relationship to meat discoloration during post-mortem storage analyzed by labelfree mass spectrometry. J Proteomics 2017;154:85-93.

22. Ryu YC, Lee MH, Lee SK, et al. Effects of muscle mass and fiber type composition of longissimus dorsi muscle on postmortem metabolic rate and meat quality in pigs. J Muscle Foods 2006;17:343-53.

23. Jeong DW, Choi YM, Lee SH, et al. Correlations of trained panel sensory values of cooked pork with fatty acid composition, muscle fiber type, and pork quality characteristics in Berkshire pigs. Meat Sci 2010;86:607-15.

24. Gondret F, Lefaucheur L, Juin H, et al. Low birth weight is associated with enlarged muscle fiber area and impaired meat tenderness of the longissimus muscle in pigs. J Anim Sci 2006;84: 93-103.

25. Schiaffino S, Reggiani C. Molecular diversity of myofibrillar proteins: gene regulation and functional significance. Physiol Rev 1996;76:371-423.

26. Kim GD, Jeong JY, Hur SJ, et al. The relationship between meat color $\left(\mathrm{CIE} \mathrm{L}^{\star}\right.$ and $\left.\mathrm{a}^{*}\right)$, myoglobin content, and their influence on muscle fiber characteristics and pork quality. Korean J Food
Sci Anim Resour 2010;30:626-33.

27. Cleveland BD, Bower CG, Redfield AL, et al. Effect of feeding distiller's grains and supplementing with dietary antioxidants on ground beef color during retail display. Meat Sci 2015;101: 110 .

28. Korn KT, Lemenager RP, Claeys MC, et al. Supplemental vitamin D3 and zilpaterol hydrochloride. II. Effect on calcium concentration, muscle fiber type, and calpain gene expression of feedlot steers. J Anim Sci 2013;91:3332-40.

29. Hu R, Wang Z, Peng Q, et al. Effects of ghrp-2 and cysteamine administration on growth performance, somatotropic axis hormone and muscle protein deposition in yaks (Bos grunniens) with growth retardation. Plos One 2016;11:e0149461.

30. Kamalakar RB, Chiba LI, Divakala KC, et al. Effect of the degree and duration of early dietary amino acid restrictions on subsequent and overall pig performance and physical and sensory characteristics of pork. J Anim Sci 2009;87:3596-606.

31. Liu G, Wei Y, Wang Z, et al. Effects of dietary supplementation with cysteamine on growth hormone receptor and insulinlike growth factor system in finishing pigs. J Agric Food Chem 2008;56:5422-7.

32. Zhang S, Qiao S, Ren M, et al. Supplementation with branchedchain amino acids to a low-protein diet regulates intestinal expression of amino acid and peptide transporters in weanling pigs. Amino Acids 2013;45:1191-205.

33. Laspiur JP, Burton JL, Weber PSD, et al. Dietary protein intake and stage of lactation differentially modulate amino acid transporter mrna abundance in porcine mammary tissue. J Nutr 2009;139:1677-84.

34. Wu G, Bazer FW, Satterfield MC, et al. Impacts of arginine nutrition on embryonic and fetal development in mammals. Amino Acids 2013;45:241-56.

35. He L, Yang H, Hou Y, et al. Effects of dietary L-lysine intake on the intestinal mucosa and expression of cat genes in weaned piglets. Amino Acids 2013;45:383-91.

36. Fuchs BC, Bode BP. Amino acid transporters asct2 and lat1 in cancer: partners in crime? Semin Cancer Biol 2005;15:25466.

37. Verrey F, Closs EI, Wagner CA, et al. CATs and HATs: the SLC7 family of amino acid transporters. Pflügers Arch 2004;447:53242.

38. Mcleod KR, Harmon DL, Schillo KK, et al. Cysteamine-induced depletion of somatostatin in sheep: time course of depletion and changes in plasma metabolites, insulin, and growth hormone. J Anim Sci 1995;73:77-87. 